\title{
Association between post-traumatic stress disorder symptoms and bone fractures after the Great East Japan Earthquake in older adults: a prospective cohort study from the Fukushima Health Management Survey
}

Fumikazu Hayashi ${ }^{1,2^{*}}$ (D), Tetsuya Ohira ${ }^{1,2}$, Hironori Nakano ${ }^{1,2}$, Masanori Nagao $^{1,2}$, Kanako Okazaki $^{2}$, Mayumi Harigane ${ }^{2,3}$, Seiji Yasumura ${ }^{2,3}$, Masaharu Maeda ${ }^{2,4}$, Atsushi Takahashi ${ }^{2,5}$, Hirooki Yabe ${ }^{2,6}$, Yuriko Suzuki ${ }^{7}$, Kenji Kamiya ${ }^{2}$ and the Fukushima Health Management Survey Group

\begin{abstract}
Background: It has been reported that psychological stress affects bone metabolism and increases the risk of fracture. However, the relationship between bone fractures and post-traumatic stress disorder (PTSD) is unclear. This study aimed to evaluate the effects of disaster-induced PTSD symptoms on fracture risk in older adults.

Methods: This study evaluated responses from 17,474 individuals aged $\geq 65$ years without a history of fractures during the Great East Japan Earthquake who answered the Mental Health and Lifestyle Survey component of the Fukushima Health Management Survey conducted in 2011. The obtained data could determine the presence or absence of fractures until 2016. Age, sex, physical factors, social factors, psychological factors, and lifestyle factors were subsequently analyzed. Survival analysis was then performed to determine the relationship between the fractures and each factor. Thereafter, univariate and multivariate Cox proportional hazard models were constructed to identify fracture risk factors.

(Continued on next page)
\end{abstract}

\footnotetext{
* Correspondence: fhayashi@fmu.ac.jp

'Department, of Epidemiology, School of Medicine, Fukushima Medical

University, 1 Hikarigaoka, Fukushima-city, Fukushima 960-1295, Japan

${ }^{2}$ Radiation Medical Science Center for the Fukushima Health Management

Survey, Fukushima Medical University, 1 Hikarigaoka, Fukushima-city,

Fukushima 960-1295, Japan

Full list of author information is available at the end of the article
}

C The Author(s). 2021 Open Access This article is licensed under a Creative Commons Attribution 4.0 International License, which permits use, sharing, adaptation, distribution and reproduction in any medium or format, as long as you give appropriate credit to the original author(s) and the source, provide a link to the Creative Commons licence, and indicate if changes were made. The images or other third party material in this article are included in the article's Creative Commons licence, unless indicated otherwise in a credit line to the material. If material is not included in the article's Creative Commons licence and your intended use is not permitted by statutory regulation or exceeds the permitted use, you will need to obtain permission directly from the copyright holder. To view a copy of this licence, visit http://creativecommons.org/licenses/by/4.0/. The Creative Commons Public Domain Dedication waiver (http://creativecommons.org/publicdomain/zero/1.0/) applies to the data made available in this article, unless otherwise stated in a credit line to the data. 


\begin{abstract}
(Continued from previous page)
Results: In total, 2,097 (12.0\%) fractures were observed throughout the follow-up period. Accordingly, univariate and multivariate Cox proportional hazard models showed that PTSD symptoms (total PTSD checklists scoring $\geq 44$ ) [hazard ratio (HR): 1.26; 95\% confidence interval (Cl): 1.10-1.44; $P=0.001$ ], history of cancer (HR: 1.49; 95\% Cl: 1.241.79; $P<0.001$ ), history of stroke (HR: 1.25; 95\% Cl: 1.03-1.52; $P=0.023$ ), history of heart disease (HR: 1.30; $95 \%$ Cl: 1.13-1.50; $P<0.001$ ), history of diabetes (HR: 1.23; 95\% Cl: 1.09-1.39; $P<0.001)$, current smoking (HR: 1.29; $95 \%$ Cl: 1.02-1.63; $P=0.036$ ), and high dissatisfaction with sleep or no sleep at all (HR: $1.33 ; 95 \% \mathrm{Cl}: 1.02-1.74 ; P=0.035$ ) promoted a significant increase in fracture risk independent of age and sex.
\end{abstract}

Conclusions: The present study indicates that disaster-induced PTSD symptoms and insomnia contribute to increased fracture risk among older adults residing in evacuation areas within the Fukushima Prefecture.

Keywords: Fractures, Mental health, Aged, Disaster victims, Fukushima nuclear accident

\section{Background}

The Great East Japan Earthquake occurred with its epicenter in the sea floor, $130 \mathrm{~km}$ off the southeast Oshika Peninsula, Miyagi Prefecture, on March 11, 2011 [1], subsequently triggering the Fukushima Daiichi Nuclear Power Station (FDNPS) accident in Fukushima Prefecture. Accordingly, the number of adult inhabitants of the evacuation zone with post-traumatic stress disorder (PTSD) checklist (PCL) scores that were above the cutoff value was comparable to that of workers affected by the 9/11 World Trade Center attack [2,3]. A survey of 240 evacuees in the evacuation area of Hirono Town, Fukushima Prefecture, revealed that 66.8 and $53.5 \%$ of evacuees had reported clinically relevant symptoms of depression and PTSD, respectively [4]. Thus, residents of evacuation areas, such as those in Fukushima Prefecture, could have presented with PTSD symptoms caused by disaster-related events.

A study recently reported a possible relationship between increased fracture risk and PTSD [5]. Therefore, residents in Fukushima evacuation areas who presented with PTSD symptoms could have also been at high risk for fractures. The results of the 2016 Basic Survey on National Life published by the Japanese Ministry of Health, Labor, and Welfare revealed that $12.1 \%$ of the 100,000 individuals requiring care had been certified as requiring support or nursing care because of falls or broken bonesmajor factors equivalent to arthritis or infirmity caused by aging [6]. Moreover, Tanji et al. reported that those with higher psychological distress after an earthquake had a higher risk for requiring nursing care than those with lower psychological distress [7]. Accordingly, the associated higher risk for increased fractures among residents in evacuation areas within Fukushima Prefecture presenting with PTSD symptoms could affect their healthy life expectancy and quality of life (QOL). In particular, the increased fracture risk among older adults could contribute to an increase in the number of those requiring support or nursing care. However, no study has examined the relationship between earthquake-induced PTSD symptoms and fractures in older adult residents of evacuation areas within Fukushima Prefecture. Therefore, investigating the association between PTSD symptoms and fractures among such residents is imperative to maintain and improve their healthy life expectancy and QOL.

We used data from the Fukushima Health Management Survey to investigate the relationship between bone fractures and PTSD symptoms after the Great East Japan Earthquake in older adults.

\section{Methods \\ Study group}

The Fukushima Health Management Survey was initiated in the Fukushima Prefecture on January 18, 2012 to investigate and observe the health status of evacuees [8]. Individuals who completed the Fukushima Health Management Survey, including the Mental Health and Lifestyle Survey component, were among those residing in any of the 13 municipalities (all areas within Hironomachi, Naraha-machi, Tomioka-machi, Kawauchi-mura, Okuma-machi, Futaba-machi, Namie-machi, Katsuraomura, and Iitate-mura, as well as parts of Tamura city, Minamisoma city, Kawamata town, and Date city) who had to be evacuated because of the Great East Japan Earthquake (registered residents) [9].

A total of 180,604 individuals aged $\geq 15$ years (born before April 1, 1995) were eligible for the 2011 edition of the registered questionnaire [10]. Valid responses with a response rate of $40.7 \%$ were obtained from 73,431 individuals (mean age: 56.4 years). After excluding 46,365 individuals aged $<65$ years, 1,220 individuals with an unknown fracture history, and 3,933 individuals who already had a history of fractures in 2011, a total of 21, 913 individuals aged $\geq 65$ years $(10,271$ men; 11,642 women; mean age: $75.0 \pm 6.9$ years) comprised the sample for the present study.

Incidences of fractures between 2012 and 2016 were determined using the questionnaire on fractures. Accordingly, 4,439 individuals were further excluded due to missing fracture data from 2012 to 2016 given that 
they had never responded to a questionnaire after 2011. Ultimately, 17,474 patients $(9,138$ women; 8,336 men; mean age, $74.3 \pm 6.5$ years; mean follow-up duration, $3.7 \pm 1.5$ person-years) were targeted (Fig. 1).

Data regarding the age, sex, physical factors (history of fractures, cancer, stroke, heart disease, diabetes, dyslipidemia, hepatic disorder, high blood pressure, and thyroid disease), social factors [experience of the earthquake, tsunami, and nuclear power plant accident (heard the explosion); need for assistance; change in employment status; and change in residence], psychological factors (history of mental illness and PCL), and lifestyle factors (history of smoking and drinking, sleep satisfaction levels, and exercise habits) obtained from the selfadministered questionnaire items used in the 2011 Mental Health and Lifestyle Survey were herein analyzed.

\section{Fracture determination}

In the Mental Health and Lifestyle Survey, questions on the presence or absence of fractures differed depending on the year. Thus, fracture incidences were determined by combining these questions.

The presence or absence of fractures in 2011 and 2012 was confirmed by responding to a question on "A history of fractures after age 50." In 2013, in addition to the aforementioned question, a combination of answers on whether "a fracture was diagnosed by a physician within the past year" was used to determine the presence or absence of fractures. In 2014 and 2015, the presence or absence of fractures was determined based on the answer to "fractures within 1 year" alone. In 2016, the incidence of fractures was determined by a question on "History of fractures after the age of 50."

\section{Definition of estimated fracture occurrence date and calculation of follow-up period}

The questionnaire used herein could not determine the date on which the fracture occurred. Therefore, this study estimated fracture occurrence dates by identifying the midpoint between the date on which the questionnaire revealed that no fracture occurred during the year and the date on which the questionnaire revealed that a fracture occurred during that year or 6 months before the date on which the questionnaire revealed that a fracture occurred during that year.

Furthermore, a number of participants also had trouble completing the questionnaire, particularly with regard to information on the month and date of completion, rendering it impossible to calculate the follow-up period. The questionnaire was distributed via mail in the month of February of the survey year (e.g., for 2011, the questionnaire was distributed in February 2012). A breakdown of the months during which the questionnaires had been filled out showed that approximately $77 \%-87 \%$ were filled out in February for each year. Therefore, for missing information of the month in which they responded, we assumed that the individuals responded in the month of February for that year. Moreover, when information regarding the date on which the

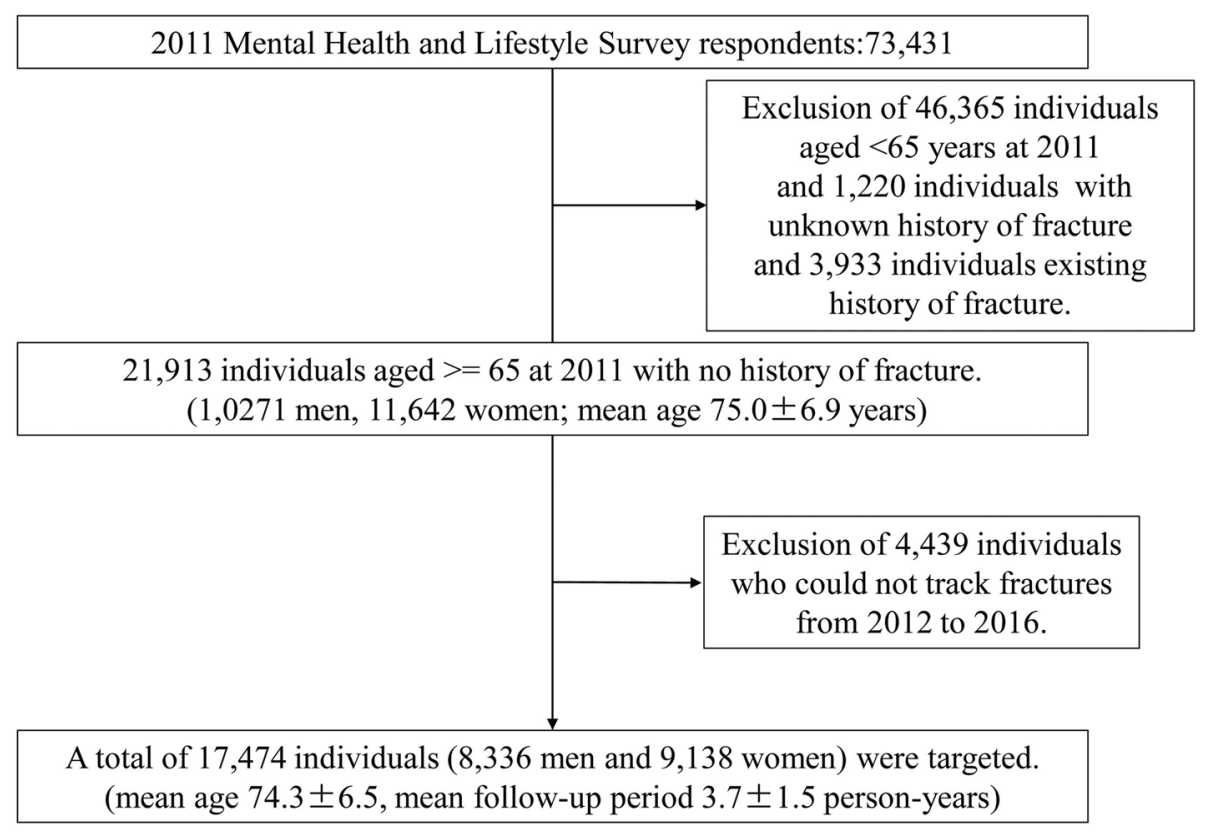

Fig. 1 Selection of study participants 
questionnaire was answered was missing, we assumed that they responded to the questionnaire on the $15^{\text {th }}$ of that month.

\section{Evaluation of post-traumatic stress disorder symptoms} The presence or absence of PTSD symptoms was evaluated using PCL [11], a self-administered questionnaire that obtained information on the symptoms of PTSD according to the diagnostic criteria in the Diagnostic and Statistical Manual of Mental Disorders-IV. The reliability, validity, and diagnostic efficiency of the Japanese version of the PCL, which was used to determine PTSD symptoms among residents who experienced the Fukushima nuclear accident, have been previously established [12]. The respondents were asked to answer a total of 17 questions using a five-point Likert scale. Accordingly, individuals with higher total scores were strongly suspected to have PTSD. Moreover, a previous study determined that a total PCL score of $\geq 44$ points was the cut-off for suspecting the presence of PTSD symptoms [13]. In this study, the total PCL score was calculated only for individuals who answered $\geq 16$ questions. When only 16 questions were answered, the average score for the 16 questions was assigned to the missing items to calculate the total score. Participants with total PCL scores $\geq 44$ were defined as those "with PTSD symptoms" and examined.

\section{History of disease}

Residents were asked whether they had a history of cancer, stroke, heart disease, hypertension, diabetes, dyslipidemia, hepatic disorder, thyroid disease, or mental illness.

\section{Lifestyle}

The questionnaire section on smoking habits had three choices: never smoked, former smoker, or current smoker. The section on drinking habits also had three choices: never drinks or rarely drinks (less than once a month), former drinker, or current drinker (more than once a month). The question on sleep satisfaction had four choices: satisfied with sleep, slightly unsatisfied with sleep, quite unsatisfied with sleep, and very dissatisfied with sleep or does not sleep at all [9]. Furthermore, the question on exercise habits had four choices: almost daily, two to four times a week, approximately once a week, or almost never.

\section{Experience of the Great East Japan Earthquake}

In the questionnaire on the experience of the Great East Japan Earthquake, individuals responded to whether they had experienced the earthquake, tsunami, and nuclear power plant accident (heard the explosion).

\section{Need for assistance}

In the question on need for assistance, individuals responded to whether they could eat, change clothes, use restrooms, and shop independently. Individuals who answered that assistance was required for any of the aforementioned four items were defined as those requiring assistance.

\section{Changes in employment status}

Residents could respond with "changed" or "unchanged" with regard to change in employment status (job change or unemployment) following the earthquake and accident [9].

\section{Changes in housing and evacuation}

Based on the question on change in residence [9], residents who lived in temporary or evacuation shelters immediately after the earthquake were defined as those who changed their residence.

Furthermore, residents of Tamura city, Minamisoma city, Date city, and Kawamata town who did not reside in a temporary or evacuation center in 2011 were defined as those who were not evacuated. Others were defined as those who were evacuated.

\section{Statistical analysis methods}

All statistical analyses were performed using SAS 9.4 (SAS Institute Inc., Cary, NC, USA). The Kaplan-Meier method and log-rank test were used to compare difference in the incidence of fractures based on questionnaire responses. Moreover, univariate and multivariate Cox proportional hazards models were used to obtain crude and adjusted hazard ratios (HRs) and 95\% confidence intervals (CIs) for the association between each factor and fractures. Furthermore, multivariate Cox proportional hazards models for men and women were established to determine differences according to sex.

In this study, it is necessary to consider the possibility that differences in questioning for fractures in each year and incomplete tracking may introduce selection and information bias. Therefore, as a sensitivity analysis, we confirmed the robustness of the results by performing a Cox regression analysis limited to individuals without missing data in the fracture questionnaire for all years.

All data are presented as number of individuals (n), mean, standard deviation, median, $25^{\text {th }}$ percentile, $75^{\text {th }}$ percentile, or percentages. $P<0.05$ indicated statistical significance.

\section{Results}

Participant characteristics

Table 1 summarizes the participants' characteristics. A total of 2,097 (12.0\%) participants experienced a 
Table 1 The association between fracture and mental health and lifestyle survey items

\begin{tabular}{|c|c|c|c|c|c|c|c|c|}
\hline \multirow[t]{2}{*}{ Factor } & \multirow[t]{2}{*}{ Classification } & \multicolumn{2}{|c|}{$\begin{array}{l}\text { All participants } \\
(n=17,474)\end{array}$} & \multicolumn{2}{|c|}{$\begin{array}{l}\text { Nonfracture group } \\
(n=15,377)\end{array}$} & \multicolumn{2}{|c|}{$\begin{array}{l}\text { Fracture group } \\
(n=2,097)\end{array}$} & \multirow[t]{2}{*}{$\begin{array}{l}P \\
\text { value }\end{array}$} \\
\hline & & Mean & SD & Mean & SD & Mean & SD & \\
\hline Age & Years & 74.3 & 6.5 & 74.2 & 6.5 & 75.5 & 6.7 & \\
\hline \multirow[t]{2}{*}{ Follow-up period } & Person-years & 3.7 & 1.5 & 3.9 & 1.4 & 2.0 & 1.4 & \\
\hline & & $\mathrm{n}$ & $\%$ & $n$ & $\%$ & $n$ & $\%$ & \\
\hline \multirow[t]{2}{*}{ Sex } & Men & 8,336 & 47.7 & 7,550 & 49.1 & 786 & 37.5 & $<0.001$ \\
\hline & Women & 9,138 & 52.3 & 7,827 & 50.9 & 1,311 & 62.5 & \\
\hline \multirow[t]{2}{*}{ PTSD symptoms } & No & 11,688 & 74.5 & 10,451 & 75.3 & 1,237 & 67.9 & $<0.001$ \\
\hline & Yes & 4,009 & 25.5 & 3,423 & 24.7 & 586 & 32.1 & \\
\hline \multirow[t]{2}{*}{ Experience of evacuation } & No & 9,382 & 54.0 & 8,298 & 54.0 & 1,084 & 51.7 & 0.228 \\
\hline & Yes & 8,092 & 46.0 & 7,079 & 46.0 & 1,013 & 48.3 & \\
\hline \multirow[t]{2}{*}{ Experience of earthquake } & No & 1,238 & 7.1 & 1,072 & 7.0 & 166 & 7.9 & 0.017 \\
\hline & Yes & 16,236 & 92.9 & 14,305 & 93.0 & 1,931 & 92.1 & \\
\hline \multirow[t]{2}{*}{ Experience of tsunami } & No & 13,452 & 77.0 & 11,821 & 76.9 & 1,631 & 77.8 & 0.309 \\
\hline & Yes & 4,022 & 23.0 & 3,556 & 23.1 & 466 & 22.2 & \\
\hline \multirow{2}{*}{$\begin{array}{l}\text { Experience of nuclear accident } \\
\text { (explosion heard) }\end{array}$} & No & 6,941 & 39.7 & 6,088 & 39.6 & 853 & 40.7 & 0.169 \\
\hline & Yes & 10,533 & 60.3 & 9,289 & 60.4 & 1,44 & 59.3 & \\
\hline \multirow[t]{2}{*}{ History of mental illness } & No & 16,066 & 94.8 & 14,170 & 95.0 & 1,896 & 93.4 & $<0.001$ \\
\hline & Yes & 875 & 5.2 & 741 & 5.0 & 134 & 6.6 & \\
\hline \multirow[t]{2}{*}{ Need for assistance } & No & 15,541 & 91.3 & 13,745 & 91.7 & 1,796 & 88.2 & $<0.001$ \\
\hline & Yes & 1,489 & 8.7 & 1,249 & 8.3 & 240 & 11.8 & \\
\hline \multirow[t]{2}{*}{ History of cancer } & No & 15,243 & 91.8 & 13,450 & 92.1 & 1,793 & 90.0 & $<0.001$ \\
\hline & Yes & 1,356 & 8.2 & 1,156 & 7.9 & 200 & 10.0 & \\
\hline \multirow[t]{2}{*}{ History of stroke } & No & 15,055 & 89.9 & 13,287 & 90.3 & 1,768 & 87.4 & $<0.001$ \\
\hline & Yes & 1,688 & 10.1 & 1,434 & 9.7 & 254 & 12.6 & \\
\hline \multirow[t]{2}{*}{ History of heart disease } & No & 13,613 & 81.0 & 12,068 & 81.7 & 1,545 & 76.6 & $<0.001$ \\
\hline & Yes & 3,185 & 19.0 & 2,712 & 18.3 & 473 & 23.4 & \\
\hline \multirow[t]{2}{*}{ History of diabetes mellitus } & No & 10,969 & 65.9 & 9,695 & 66.2 & 1,274 & 63.4 & 0.004 \\
\hline & Yes & 5,676 & 34.1 & 4,942 & 33.8 & 734 & 36.6 & \\
\hline \multirow[t]{2}{*}{ History of dyslipidemia } & No & 8,316 & 49.6 & 7,336 & 49.7 & 980 & 48.6 & 0.723 \\
\hline & Yes & 8,459 & 50.4 & 7,421 & 50.3 & 1,038 & 51.4 & \\
\hline \multirow[t]{2}{*}{ History of hepatic disorder } & No & 16,130 & 96.7 & 14,200 & 96.8 & 1,930 & 96.2 & 0.069 \\
\hline & Yes & 545 & 3.3 & 469 & 3.2 & 76 & 3.8 & \\
\hline \multirow[t]{2}{*}{ History of hypertension } & No & 4,986 & 29.1 & 4,405 & 29.3 & 581 & 28.2 & 0.182 \\
\hline & Yes & 12,123 & 70.9 & 10,644 & 70.7 & 1,479 & 71.8 & \\
\hline \multirow[t]{2}{*}{ History of thyroid disease } & No & 16,611 & 97.1 & 14,632 & 97.1 & 1,979 & 96.8 & 0.333 \\
\hline & Yes & 497 & 2.9 & 431 & 2.9 & 66 & 3.2 & \\
\hline Smoking habit & Never smoked & 10,187 & 61.3 & 8,866 & 60.5 & 1,321 & 67.3 & $<0.001$ \\
\hline & Former smoker & 4,782 & 28.8 & 4,317 & 29.5 & 465 & 23.7 & \\
\hline & Current smoker & 1,644 & 9.9 & 1,468 & 10.0 & 176 & 9.0 & \\
\hline Drinking habit & $\begin{array}{l}\text { Never drinks or rarely drinks } \\
\text { (less than once a month) }\end{array}$ & 9,307 & 55.2 & 8,121 & 54.6 & 1,186 & 59.3 & $<0.001$ \\
\hline & Former drinker & 958 & 5.7 & 847 & 5.7 & 111 & 5.6 & \\
\hline & $\begin{array}{l}\text { Current drinker } \\
\text { (more than once a month) }\end{array}$ & 6,596 & 39.1 & 5,893 & 39.7 & 703 & 35.2 & \\
\hline
\end{tabular}


Table 1 The association between fracture and mental health and lifestyle survey items (Continued)

\begin{tabular}{|c|c|c|c|c|c|c|c|c|}
\hline \multirow[t]{2}{*}{ Factor } & \multirow[t]{2}{*}{ Classification } & \multicolumn{2}{|c|}{$\begin{array}{l}\text { All participants } \\
(n=17,474)\end{array}$} & \multicolumn{2}{|c|}{$\begin{array}{l}\text { Nonfracture group } \\
(n=15,377)\end{array}$} & \multicolumn{2}{|c|}{$\begin{array}{l}\text { Fracture group } \\
(n=2,097)\end{array}$} & \multirow[t]{2}{*}{$\begin{array}{l}P \\
\text { value }\end{array}$} \\
\hline & & Mean & SD & Mean & SD & Mean & SD & \\
\hline \multirow[t]{4}{*}{ Level of sleep satisfaction } & Satisfied with sleep & 5,309 & 41.9 & 4,738 & 42.6 & 571 & 36.9 & $<0.001$ \\
\hline & Slightly unsatisfied with sleep & 5,122 & 40.4 & 4,488 & 40.4 & 634 & 41.0 & \\
\hline & Quite unsatisfied with sleep & 1,650 & 13.0 & 1,408 & 12.7 & 242 & 15.6 & \\
\hline & $\begin{array}{l}\text { Very dissatisfied with sleep or does } \\
\text { not sleep at all }\end{array}$ & 583 & 4.6 & 483 & 4.3 & 100 & 6.5 & \\
\hline \multirow[t]{4}{*}{ Exercise habit } & Almost daily & 4,303 & 25.9 & 3,829 & 26.2 & 474 & 23.9 & 0.003 \\
\hline & 2 to 4 times a week & 5,231 & 31.5 & 4,620 & 31.6 & 611 & 30.8 & \\
\hline & Approximately once a week & 2,505 & 15.1 & 2,193 & 15.0 & 312 & 15.7 & \\
\hline & Almost never & 4,586 & 27.6 & 4,000 & 27.3 & 586 & 29.6 & \\
\hline \multirow[t]{2}{*}{ Job change } & No & 8,242 & 54.5 & 7,297 & 54.6 & 945 & 53.7 & 0.884 \\
\hline & Yes & 6,874 & 45.5 & 6,058 & 45.4 & 816 & 46.3 & \\
\hline \multirow[t]{2}{*}{ Loss of job } & No & 15,132 & 86.6 & 13,307 & 86.5 & 1,825 & 87.0 & 0.145 \\
\hline & Yes & 2,342 & 13.4 & 2,070 & 13.5 & 272 & 13.0 & \\
\hline \multirow[t]{2}{*}{ Residential changes } & No & 10,355 & 62.1 & 9,135 & 62.2 & 1,220 & 61.4 & 0.738 \\
\hline & Yes & 6,324 & 37.9 & 5,557 & 37.8 & 767 & 38.6 & \\
\hline
\end{tabular}

Data are presented as a number with a percentage or a mean with standard deviation

The interval scale between the bone fracture and no bone fracture group groups was tested using the log-rank test

$S D$ standard deviation, PTSD post-traumatic stress disorder

$p<0.05$ was considered statistically significant

fracture during the follow-up period, with an incidence rate of $0.032 /$ year.

\section{Survival analysis results}

The relationship between each factor and the incidence of fractures was examined among participants divided into the fracture and nonfracture groups (Table 1). Accordingly, survival analysis results found significant differences in fracture incidence among older adults according to sex $(P<0.001)$, PTSD symptoms $(P<$ $0.001)$, experience of earthquake $(P=0.017)$, history of mental illness $(P<0.001)$, need for assistance $(P<0.001)$, history of cancer $(P<0.001)$, history of stroke $(P<$ $0.001)$, history of heart disease $(P<0.001)$, history of diabetes $(P=0.004)$, smoking habits $(P<0.001)$, drinking habits $(P<0.001)$, sleep satisfaction $(P<0.001)$, and exercise habits $(P=0.003)$.

\section{Univariate and multivariate Cox proportional hazards models}

Univariate and multivariate Cox proportional hazards models were established using factors that were determined to be significant during survival analysis to identify the association between psychological indicators and fracture frequency among older adults (Table 2). Accordingly, the multivariate and univariate Cox proportional hazards analyses showed that PTSD symptoms (HR: $1.26 ; 95 \%$ CI: $1.10-1.44 ; P=0.001$ ), history of cancer (HR: 1.49; 95\% CI: 1.24-1.79; $P<0.001$ ), history of stroke (HR: 1.25 ; 95\% CI: $1.03-1.52$; $P=0.023$ ), history of heart disease (HR: 1.30; 95\% CI: 1.13-1.50; $P<$ 0.001 ), history of diabetes (HR: 1.23 ; 95\% CI: $1.09-1.39$; $P<0.001$ ), current smoking(HR: 1.29 ; 95\% CI: 1.02-1.63; $P=0.036)$, and high dissatisfaction with sleep or no sleep at all (HR: 1.33 ; 95\% CI: $1.02-1.74 ; P=0.035$ ) significantly increased fracture risk, independent of age and sex.

Table 3 presents the results of multivariate Cox proportional hazards analysis according to sex to determine the sex-related differences. Accordingly, PTSD symptoms (HR: $1.39 ; 95 \% \mathrm{CI}: 1.11-1.74 ; P=0.004$ ), history of cancer (HR: $1.50 ; 95 \%$ CI: $1.16-1.95 ; P=0.002$ ), history of diabetes (HR: 1.35 ; 95\% CI: $1.12-1.63 ; P=0.001$ ), and high dissatisfaction with sleep or no sleep at all (HR: 1.71; 95\% CI: $1.12-2.60 ; P=0.013$ ) had significantly increased fracture risk among older men, independent of age. In contrast, histories of cancer (HR: 1.45; 95\% CI: 1.11-1.90; $P=0.007$ ) and heart disease (HR: 1.36; $95 \%$ CI: $1.13-1.64 ; P=0.001)$ significantly increased fracture risk among older women, independent of age. The primary conclusions obtained herein remained largely the same regardless of whether the entry date was supplied.

\section{Sensitivity analysis}

In this study, it is important to consider the possibility that differences in questions for fractures in each year 
Table 2 The results of univariate and multivariate Cox proportional hazard models

\begin{tabular}{|c|c|c|c|c|c|c|c|}
\hline Factors & Classification & $\begin{array}{l}\text { Nonfracture } \\
\text { group } \\
(n=15,377)\end{array}$ & $\begin{array}{l}\text { Fracture } \\
\text { group } \\
(n=2,097)\end{array}$ & $\begin{array}{l}\text { Crude HR } \\
(95 \% \mathrm{Cl})\end{array}$ & $\begin{array}{l}P \\
\text { value }\end{array}$ & $\begin{array}{l}\text { Adjusted HR }(95 \% \\
\mathrm{Cl})^{\mathrm{a}} \\
(n=10,032)\end{array}$ & $P$ value \\
\hline Age & Continuous & & & $1.04(1.04-1.05)$ & $<0.001$ & $1.04(1.03-1.05)$ & $<0.001$ \\
\hline \multirow[t]{2}{*}{ Sex } & Men & 7,550 & 786 & Ref & & Ref & \\
\hline & Women & 7,827 & 1,311 & $1.59(1.45-1.73)$ & $<0.001$ & $1.85(1.55-2.20)$ & $<0.001$ \\
\hline \multirow[t]{2}{*}{ PTSD symptoms } & No & 10,451 & 1,237 & Ref & & Ref & \\
\hline & Yes & 3,423 & 586 & $1.43(1.30-1.58)$ & $<0.001$ & $1.26(1.10-1.44)$ & 0.001 \\
\hline \multirow{2}{*}{$\begin{array}{l}\text { Experience of } \\
\text { earthquake }\end{array}$} & No & 1,072 & 166 & Ref & & Ref & \\
\hline & Yes & 14,305 & 1,931 & $0.82(0.70-0.97)$ & 0.017 & $0.92(0.69-1.20)$ & 0.531 \\
\hline \multirow{2}{*}{$\begin{array}{l}\text { History of mental } \\
\text { illness }\end{array}$} & No & 14,170 & 1,896 & Ref & & Ref & \\
\hline & Yes & 741 & 134 & $1.45(1.21-1.72)$ & $<0.001$ & $0.98(0.76-1.27)$ & 0.869 \\
\hline \multirow{2}{*}{$\begin{array}{l}\text { Need for } \\
\text { assistance }\end{array}$} & No & 13,745 & 1,796 & Ref & & Ref & \\
\hline & Yes & 1,249 & 240 & $1.85(1.62-2.12)$ & $<0.001$ & $1.14(0.92-1.41)$ & 0.240 \\
\hline \multirow[t]{2}{*}{ History of cancer } & No & 13,745 & 1,796 & Ref & & Ref & \\
\hline & Yes & 1,249 & 240 & $1.31(1.13-1.51)$ & $<0.001$ & $1.49(1.24-1.79)$ & $<0.001$ \\
\hline \multirow[t]{2}{*}{ History of stroke } & No & 13,287 & 1,768 & Ref & & Ref & \\
\hline & Yes & 1,434 & 254 & $1.41(1.24-1.61)$ & $<0.001$ & $1.25(1.03-1.52)$ & 0.023 \\
\hline \multirow{2}{*}{$\begin{array}{l}\text { History of heart } \\
\text { disease }\end{array}$} & No & 12,068 & 1,545 & Ref & & Ref & \\
\hline & Yes & 2,712 & 473 & $1.37(1.23-1.51)$ & $<0.001$ & $1.30(1.13-1.50)$ & $<0.001$ \\
\hline \multirow{2}{*}{$\begin{array}{l}\text { History of diabetes } \\
\text { mellitus }\end{array}$} & No & 9,695 & 1,274 & Ref & & Ref & \\
\hline & Yes & 4,942 & 734 & $1.14(1.04-1.25)$ & 0.004 & $1.23(1.09-1.39)$ & $<0.001$ \\
\hline \multirow[t]{3}{*}{ Smoking habit } & Never smoked & 8,866 & 1,321 & Ref & & Ref & (trend $p=0.057$ ) \\
\hline & Former smoker & 4,317 & 465 & $0.73(0.66-0.81)$ & $<0.001$ & $1.03(0.86-1.24)$ & 0.739 \\
\hline & Current smoker & 1,468 & 176 & $0.83(0.71-0.97)$ & 0.022 & $1.29(1.02-1.63)$ & 0.036 \\
\hline \multirow[t]{3}{*}{ Drinking habit } & $\begin{array}{l}\text { Never drinks or rarely drinks } \\
\text { (less than once a month) }\end{array}$ & 8,121 & 1,186 & Ref & & Ref & (trend $p=0.134$ ) \\
\hline & Former drinker & 847 & 111 & $0.95(0.78-1.15)$ & 0.595 & $1.22(0.92-1.63)$ & 0.171 \\
\hline & $\begin{array}{l}\text { Current drinker (more than } \\
\text { once a month) }\end{array}$ & 5,893 & 703 & $0.79(0.72-0.87)$ & $<0.001$ & $1.13(0.98-1.31)$ & 0.105 \\
\hline \multirow{4}{*}{$\begin{array}{l}\text { Level of sleep } \\
\text { satisfaction }\end{array}$} & Satisfied with sleep & 4,738 & 571 & Ref & & Ref & (trend $p=0.142$ ) \\
\hline & $\begin{array}{l}\text { Slightly unsatisfied with } \\
\text { sleep }\end{array}$ & 4,488 & 634 & $1.15(1.02-1.28)$ & 0.018 & $1.04(0.92-1.19)$ & 0.552 \\
\hline & Quite unsatisfied with sleep & 1,408 & 242 & $1.40(1.20-1.62)$ & $<0.001$ & $1.03(0.85-1.24)$ & 0.796 \\
\hline & $\begin{array}{l}\text { Very dissatisfied with sleep } \\
\text { or does not sleep at all }\end{array}$ & 483 & 100 & $1.69(1.37-2.09)$ & $<0.001$ & $1.33(1.02-1.74)$ & 0.035 \\
\hline \multirow[t]{4}{*}{ Exercise habit } & Almost daily & 3,829 & 474 & Ref & & Ref & (trend $p=0.359$ ) \\
\hline & 2 to 4 times a week & 4,620 & 611 & $1.06(0.94-1.20)$ & 0.338 & $1.00(0.85-1.17)$ & 0.976 \\
\hline & $\begin{array}{l}\text { Approximately once a } \\
\text { week }\end{array}$ & 2,193 & 312 & $1.16(1.00-1.33)$ & 0.047 & $1.08(0.89-1.30)$ & 0.443 \\
\hline & Almost never & 4,000 & 586 & $1.24(1.10-1.40)$ & $<0.001$ & $1.06(0.90-1.25)$ & 0.480 \\
\hline
\end{tabular}

${ }^{a}$ Adjusted for age, sex, PCL score, experience of earthquake, history of mental illness, need for assistance, history of cancer, history of stroke, history of heart disease, history of diabetes mellitus, smoking habit, drinking habit, level of sleep satisfaction, and exercise habit. 95\% Cl 95\% confidence interval, $H R$ hazard ratio, Ref reference, PTSD post-traumatic stress disorder. Cox proportional hazard model; $p<0.05$ was considered statistically significant

and incomplete tracking may introduce selection and information bias. Therefore, as a sensitivity analysis, we performed a multivariate Cox regression analysis limited to individuals without missing information in the fracture questionnaire for all years $(n=3,129)$ (Table 4$)$. The results showed that the HRs of PTSD symptoms for individuals with fractures were similar to those presented in Table 2, indicating the robustness of the results in Table 2. 
Table 3 The results of multivariate Cox proportional hazard models by sex

\begin{tabular}{|c|c|c|c|c|c|}
\hline \multirow[t]{2}{*}{ Factors } & \multirow[t]{2}{*}{ Classification } & \multicolumn{2}{|l|}{ Men $(n=5,185)$} & \multicolumn{2}{|l|}{ Women $(n=4,847)$} \\
\hline & & $\begin{array}{l}\text { Adjusted HR (95\% } \\
\mathrm{Cl}^{\mathrm{a}}\end{array}$ & $P$ value & $\begin{array}{l}\text { Adjusted HR (95\% } \\
\mathrm{Cl})^{\mathrm{b}}\end{array}$ & $P$ value \\
\hline Age & Continuous & $1.03(1.02-1.05)$ & $<0.001$ & $1.04(1.03-1.06)$ & $<0.001$ \\
\hline \multirow[t]{2}{*}{ PTSD symptoms } & No & Ref & & Ref & \\
\hline & Yes & $1.39(1.11-1.74)$ & 0.004 & $1.18(0.99-1.41)$ & 0.067 \\
\hline \multirow{2}{*}{$\begin{array}{l}\text { Experience of } \\
\text { earthquake }\end{array}$} & No & Ref & & Ref & \\
\hline & Yes & $0.86(0.59-1.25)$ & 0.433 & $0.92(0.62-1.38)$ & 0.698 \\
\hline \multirow{2}{*}{$\begin{array}{l}\text { History of mental } \\
\text { illness }\end{array}$} & No & Ref & & Ref & \\
\hline & Yes & $0.90(0.58-1.38)$ & 0.646 & $1.02(0.74-1.40)$ & 0.906 \\
\hline \multirow[t]{2}{*}{ Need for assistance } & No & Ref & & Ref & \\
\hline & Yes & $0.94(0.61-1.43)$ & 0.765 & $1.21(0.94-1.56)$ & 0.140 \\
\hline \multirow[t]{2}{*}{ History of cancer } & No & Ref & & Ref & \\
\hline & Yes & $1.50(1.16-1.95)$ & 0.002 & $1.45(1.11-1.90)$ & 0.007 \\
\hline \multirow[t]{2}{*}{ History of stroke } & No & Ref & & Ref & \\
\hline & Yes & $1.29(0.99-1.69)$ & 0.061 & $1.18(0.89-1.57)$ & 0.253 \\
\hline \multirow{2}{*}{$\begin{array}{l}\text { History of heart } \\
\text { disease }\end{array}$} & No & Ref & & Ref & \\
\hline & Yes & $1.21(0.97-1.50)$ & 0.082 & $1.36(1.13-1.64)$ & 0.001 \\
\hline \multirow{2}{*}{$\begin{array}{l}\text { History of diabetes } \\
\text { mellitus }\end{array}$} & No & Ref & & Ref & \\
\hline & Yes & $1.35(1.12-1.63)$ & 0.001 & $1.14(0.97-1.34)$ & 0.122 \\
\hline \multirow[t]{3}{*}{ Smoking habit } & Never smoked & Ref & (trend $p=0.200$ ) & Ref & (trend $p=0.116$ ) \\
\hline & Former smoker & $1.02(0.82-1.26)$ & 0.869 & $1.10(0.75-1.62)$ & 0.612 \\
\hline & Current smoker & $1.22(0.92-1.62)$ & 0.166 & $1.46(0.95-2.28)$ & 0.087 \\
\hline \multirow[t]{3}{*}{ Drinking habit } & $\begin{array}{l}\text { Never drinks or rarely drinks (less than once } \\
\text { a month) }\end{array}$ & Ref & (trend $p=0.425)$ & Ref & (trend $p=0.210$ ) \\
\hline & Former drinker & $1.28(0.92-1.77)$ & 0.141 & $0.90(0.40-2.04)$ & 0.798 \\
\hline & Current drinker (more than once a month) & $1.13(0.90-1.40)$ & 0.293 & $1.15(0.93-1.41)$ & 0.193 \\
\hline \multirow{4}{*}{$\begin{array}{l}\text { Level of sleep } \\
\text { satisfaction }\end{array}$} & Satisfied with sleep & Ref & (trend $p=0.029$ ) & Ref & (trend $p=0.972$ ) \\
\hline & Slightly unsatisfied with sleep & $0.99(0.80-1.21)$ & 0.986 & $1.06(0.90-1.26)$ & 0.493 \\
\hline & Quite unsatisfied with sleep & $1.26(0.94-1.69)$ & 0.126 & $0.90(0.70-1.16)$ & 0.409 \\
\hline & $\begin{array}{l}\text { Very dissatisfied with sleep or does not } \\
\text { sleep at all }\end{array}$ & $1.71(1.12-2.60)$ & 0.013 & $1.14(0.81-1.62)$ & 0.454 \\
\hline \multirow[t]{4}{*}{ Exercise habit } & Almost daily & Ref & (trend $p=0.819$ ) & Ref & (trend $p=0.310$ ) \\
\hline & 2 to 4 times a week & $0.92(0.73-1.17)$ & 0.503 & $1.07(0.86-1.33)$ & 0.530 \\
\hline & About once a week & $0.99(0.74-1.33)$ & 0.955 & $1.17(0.90-1.51)$ & 0.240 \\
\hline & Almost never & $1.01(0.78-1.29)$ & 0.966 & $1.12(0.90-1.40)$ & 0.321 \\
\hline
\end{tabular}

$\overline{a, b}$ Adjusted for age, PCL score, experience of earthquake, history of mental illness, need for assistance, history of cancer, history of stroke, history of heart disease, history of diabetes mellitus, smoking habit, drinking habit, level of sleep satisfaction, and exercise habit. $95 \% \mathrm{Cl} 95 \%$ confidence interval, $\mathrm{HR}$ hazard ratio, Ref reference, PTSD post-traumatic stress disorder. Cox proportional hazard model; $p<0.05$ was considered statistically significant

\section{Discussion}

Our study suggested that PTSD symptoms were significantly associated with the occurrence of fractures among older adults, particularly men, who resided in evacuation areas within Fukushima Prefecture. Studies have reported an increase in the prevalence of diseases, such as obesity and lifestyle-related diseases, in the residents of evacuation areas within Fukushima Prefecture [14-17].
This increase in disease prevalence could be partially attributed to the increase in stress caused by environmental changes due to moving into temporary housing, living in an evacuation site outside the local area, or disturbance in eating habits [18-21]. Thus, psychological stress has been considered to be associated with adverse health effects among residents of evacuation areas throughout Fukushima Prefecture. A study found that 
Table 4 Sensitivity analysis results of participants who responded to all surveys from 2011 to 2016

\begin{tabular}{|c|c|c|c|}
\hline Factors & Classification & $\begin{array}{l}\text { Adjusted HR }(95 \% \mathrm{Cl})^{\mathrm{a}} \\
(n=3,129)\end{array}$ & $P$ value \\
\hline Age & Continuous & $1.05(1.03-1.07)$ & $<0.001$ \\
\hline \multirow[t]{2}{*}{ Sex } & Men & Ref & \\
\hline & Women & $2.09(1.55-2.83)$ & $<0.001$ \\
\hline \multirow[t]{2}{*}{ PTSD symptoms } & No & Ref & \\
\hline & Yes & $1.26(0.99-1.59)$ & 0.063 \\
\hline \multirow[t]{2}{*}{ Experience of earthquake } & No & Ref & \\
\hline & Yes & $0.99(0.61-1.62)$ & 0.982 \\
\hline \multirow[t]{2}{*}{ History of mental illness } & No & Ref & \\
\hline & Yes & $0.78(0.47-1.31)$ & 0.354 \\
\hline \multirow[t]{2}{*}{ Need for assistance } & No & Ref & \\
\hline & Yes & $0.89(0.54-1.47)$ & 0.642 \\
\hline \multirow[t]{2}{*}{ History of cancer } & No & Ref & \\
\hline & Yes & $1.71(1.26-2.32)$ & $<0.001$ \\
\hline \multirow[t]{2}{*}{ History of stroke } & No & Ref & \\
\hline & Yes & $1.34(0.95-1.89)$ & 0.098 \\
\hline \multirow[t]{2}{*}{ History of heart disease } & No & Ref & \\
\hline & Yes & $1.36(1.07-1.73)$ & 0.012 \\
\hline \multirow[t]{2}{*}{ History of diabetes mellitus } & No & Ref & \\
\hline & Yes & $1.37(1.12-1.68)$ & 0.003 \\
\hline \multirow[t]{3}{*}{ Smoking habit } & Never smoked & Ref & (trend $p=0.519$ ) \\
\hline & Former smoker & $1.15(0.85-1.55)$ & 0.361 \\
\hline & Current smoker & $1.07(0.69-1.67)$ & 0.751 \\
\hline \multirow[t]{3}{*}{ Drinking habit } & Never drinks or rarely drinks (less than once a month) & Ref & (trend $p=0.159$ ) \\
\hline & Former drinker & $1.07(0.63-1.81)$ & 0.795 \\
\hline & Current drinker (more than once a month) & $1.19(0.93-1.53)$ & 0.168 \\
\hline \multirow[t]{4}{*}{ Level of sleep satisfaction } & Satisfied with sleep & Ref & (trend $p=0.429$ ) \\
\hline & Slightly unsatisfied with sleep & $1.07(0.86-1.33)$ & 0.557 \\
\hline & Quite unsatisfied with sleep & $1.02(0.73-1.41)$ & 0.924 \\
\hline & Very dissatisfied with sleep or does not sleep at all & $1.27(0.79-2.02)$ & 0.324 \\
\hline \multirow[t]{4}{*}{ Exercise habit } & Almost daily & Ref & (trend $p=0.919$ ) \\
\hline & 2 to 4 times a week & $1.12(0.87-1.44)$ & 0.374 \\
\hline & Approximately once a week & $0.87(0.62-1.21)$ & 0.413 \\
\hline & Almost never & $1.06(0.80-1.39)$ & 0.691 \\
\hline
\end{tabular}

${ }^{a}$ Adjusted for age, sex, PCL score, experience of earthquake, history of mental illness, need for assistance, history of cancer, history of stroke, history of heart disease, history of diabetes mellitus, smoking habit, drinking habit, level of sleep satisfaction, and exercise habit. $95 \% \mathrm{Cl} 95 \%$ confidence interval, $H R$ hazard ratio, Ref reference, PTSD post-traumatic stress disorder. Cox proportional hazard model; $p<0.05$ was considered statistically significant

individuals who reported to have experienced high levels of psychological stress were at increased risk for fractures caused by osteoporosis [22]. One possible mechanism underlying the association between stress and fracture risk is that psychological stress increases cortisol secretion via the hypothalamus-pituitary-adrenal system. Glucocorticoids induce bone loss and increase the risk of osteoporotic fractures [23, 24]. Individuals with
PTSD symptoms can be considered to have had high psychological stress immediately after a disaster. Furthermore, studies have reported that older adults and those living under extreme conditions were more likely to experience worse symptoms [25]. The psychological effects caused by the Fukushima nuclear accident have been widespread, causing not only trauma symptoms but also chronic and more complex social problems, 
such as stigma and community and family fragmentation [26]. Therefore, persistent high levels of stress caused by disasters could contribute to increased fracture risk in older adults. To prevent fractures after a disaster, older adults with PTSD symptoms should be assessed for bone mineral density and receive aggressive interventions to reduce psychosocial stress.

Furthermore, those with PTSD are presumed to have an increased likelihood of suffering from other mental disorders, such as depression [27, 28]. In previous studies, the percentage of residents with PCL scores above the cut-off point was significantly higher in residents with Kessler psychological distress scale (K6) scores [29-31] above the cut-off than in those with K6 score below the cut-off [32]. Furthermore, prefectural health surveys have reported that the coexistence of PTSD and previous mental illness or mental disorders were poor predictors of mid-term mental health [33]. Thus, the mental health deterioration caused by a disaster can promote even more confined and sedentary lifestyles among older adults who already tend to have low physical function in a depressed state, causing a decrease in physical function and a corresponding increased risk of fractures. Therefore, social participation should be encouraged in older adults with PTSD symptoms and low physical function to maintain and improve their physical function and mental health.

Depression itself has also been reported to be associated with an increased risk of fractures [34], which may be mediated by the use of antidepressants [35]. For instance, receiving one class of antidepressants, selective serotonin reuptake inhibitors (SSRIs), has been reported to increase the risk of fractures regardless of the presence of depression or bone density [36]. Moreover, SSRIs have been reported to contribute to fractureinduced falls and increased fracture risk [37]. Considering that SSRIs have occasionally been considered for the treatment of PTSD, older adults receiving medication for PTSD symptoms must be aware of the risk for fractures facilitated by antidepressants.

The present study found that those who were extremely dissatisfied with their sleep, particularly older men, were at increased risk of fractures. The prevalence of insomnia and use of sleeping pills among Japanese individuals have been reported to increase with age [38]. Benzodiazepines or benzodiazepine receptor agonists, a nonbenzodiazepine alternative, have been among the commonly prescribed sleeping pills in Japan. Accordingly, studies have shown that prolonged and high-dose usage of benzodiazepines was associated with an increased risk for falls and fractures [39-41], suggesting that insomnia pharmacotherapy could have also contributed toward increasing fracture risk among older adults, such as those residing in evacuation areas within
Fukushima Prefecture. Understanding the sleep environment and providing guidance on sleep hygiene should be the initial management for insomnia. Our study suggests that securing sleep time and improving sleep quality are imperative for preventing fractures among older adults, particularly men, residing in evacuation areas within Fukushima Prefecture.

Our study found that women have a higher risk for fractures than men. However, although PTSD symptoms tended to be associated with the occurrence of fractures in women, it was not statistically significant. Moreover, sleep satisfaction was not significantly associated with the occurrence of fractures in women. In women, with regard to fractures, other factors may be more influential than the prevalence of PTSD symptoms and stress from lack of sleep. Primary osteoporosis among women is often caused by heredity, aging, and postmenopausal decline in female hormones [42]. Furthermore, patients with osteoporosis have been reported to be more likely to experience fractures after a fall [43]. Osteoporosisrelated fractures can also have a significant impact on health-related QOL (HRQOL) [44]. Thus, the aforementioned results suggest that health problems that are specific to women, which could not be investigated herein, could have had a greater effect on fracture risk than the effect of increased psychological stress. However, exercise can be effective in reducing falls and risk factors associated with fractures from falls among patients with low bone mineral density [45]. Therefore, especially for women, regular bone density measurements and exercise habit formation for those with PTSD symptoms are recommended to prevent fractures from falling and a decline in HRQOL.

This study revealed that individuals who were current smokers and those with a history of diabetes, heart disease, stroke, and cancer were at an increased risk for fractures. Indeed, previous studies have reported that the prevalence of smoking habits [46], type 2 diabetes [47], cardiovascular disease [48], stroke [49], and cancer [50, $51,52]$ increased the risk for fractures, suggesting that a comprehensive strategy, including smoking cessation to prevent lifestyle-related diseases, cardiovascular events, and cancer, is necessary for preventing fractures among older adults residents of evacuation areas.

The present study has certain noteworthy limitations. First, the age-adjusted prevalence of post-traumatic stress has been known to decrease every year, whereas studies have shown that the mental health of residents in evacuation areas within Fukushima Prefecture has improved compared to that at the time of the earthquake [53]. However, whether this improvement is prevalent among residents of the 13 municipalities remains unclear given that our participants comprised only a small percentage of those who participated in the Fukushima 
Health Management Survey. Horikoshi et al. also reported that those who did not respond to the mental survey had a significantly higher rate of psychological distress than the respondents [54]. Therefore, the results of this study could have underestimated the impact of increased PTSD symptoms caused by the Great East Japan Earthquake and FDNPS accident on fractures. Accordingly, it may be necessary to expand the scope of psychological research by including a survey on the mental health of nonrespondents.

Second, this survey did not include details on the medication conditions, bone density tests, fracture sites, circumstances during which fractures sustained, presence of osteoporosis, or use of antidepressants. Hence, factors that could contribute to fracture risk, such as the prevalence of osteoporosis and use of antidepressants and steroids [55], could not be investigated. Moreover, the effects of sex differences on fractures could not be completely clarified given that information on menopause or hormone levels among women was not surveyed. Therefore, future studies should include examinations and questions addressing these factors.

Third, studies on postmenopausal women have reported that obesity and underweight were both risk factors for fractures [56]. However, given that the present survey did not include items on height and weight in FY2011, body mass index could not be calculated. Therefore, we plan to examine the relationship between weight and fractures by evaluating health checkup data in our next study.

Fourth, the results of a systematic review and metaanalysis showed that frailty and pre-frailty were significant predictors of fractures among community-dwelling older adults [57]. Frailty can be assessed using the frailty index [58], which combines several variables (functioning, cognition, comorbidities, health attitudes and habits, and physical performance). However, the Mental Health and Lifestyle Survey does not include many questions on physical functioning, whereas the present survey items did not include the association between frailty and fractures. Future investigations may need to include a questionnaire on health examination results to screen for frailty.

Fifth, certain nutrients and foods have been reported to be associated with fracture risk [59, 60]. Previous studies on the Fukushima Health Management Survey also reported an association between psychological distress and food intake [20]. However, the Fukushima Health Management Survey contained a limited number of questions on food intake. Moreover, a clear bias was noted when evaluating each food group. Accordingly, we determined that data obtained from this study did not allow a comprehensive examination of the association between fractures and food intake and unfortunately, this information was not considered.

\section{Conclusions}

The present study indicated that disaster-induced PTSD symptoms and insomnia contribute to increased fracture risk among older adults residing in evacuation areas within Fukushima Prefecture. Offering active psychological care to reduce psychosocial stress and providing guidance on sleep are important to prevent fractures in older adult residents, such as those living in evacuation areas.

\section{Abbreviations}

PTSD: Post-traumatic stress disorder; PCL: Post-traumatic stress disorder checklist; HR: Hazard ratio; Cl: Confidence interval; FDNPS: Fukushima Daiichi Nuclear Power Station; QOL: Quality of life; K6: Kessler psychological distress scale; SSRIs: Selective serotonin reuptake inhibitors; HRQOL: Health-related quality of life; FY: Fiscal year; SD: Standard deviation; Ref: Reference

\section{Acknowledgments}

The present study was conducted by the Fukushima Medical University on consignment by the Fukushima Prefecture using the Fukushima prefectural health survey funds. Furthermore, the opinions presented in the report are those of the authors and not of the Fukushima Prefecture residents.

\section{Authors' contributions}

Conception and design: FH, TO, SY, MM, HY, YS, and KK. Acquisition of data: $\mathrm{MH}, \mathrm{MM}, \mathrm{HY}$, and SY. Analysis and interpretation of data: FH. Drafting the article: FH. Revising it for intellectual content: TO, HN, MN, KO, MH, SY, MM, AT, HY, YS, and KK. Final approval of the completed article: All authors.

\section{Funding}

This survey was conducted as part of "Fukushima Prefecture's post-disaster recovery plans" and was supported by the national "Health Fund for Children and Adults Affected by the Nuclear Incident." The funding organization had no role in the design of the study, data collection, analysis, interpretation of data, or writing the manuscript.

\section{Availability of data and materials}

The datasets analyzed during the present study are not publicly available because the data of the Fukushima Health Management Survey belongs to the government of Fukushima Prefecture and can only be used within that organization.

\section{Compliance with ethical standards}

\section{Ethics approval and consent to participate}

The mental health survey participants were informed in writing that the survey results would be totaled and reported after analysis, and only those who returned the self-recorded questionnaire were considered to have provided consent to participate in the study. Furthermore, the study was approved by the ethical review board of Fukushima Medical University (approval numbers: 1316 and 2148).

\section{Consent for publication}

Not applicable.

\section{Competing interests}

The authors declare that there are no competing interests.

\section{Author details}

${ }^{1}$ Department, of Epidemiology, School of Medicine, Fukushima Medical University, 1 Hikarigaoka, Fukushima-city, Fukushima 960-1295, Japan. ${ }^{2}$ Radiation Medical Science Center for the Fukushima Health Management Survey, Fukushima Medical University, 1 Hikarigaoka, Fukushima-city, Fukushima 960-1295, Japan. ${ }^{3}$ Department of Public Health, School of Medicine, Fukushima Medical University, 1 Hikarigaoka, Fukushima-city, Fukushima 960-1295, Japan. ${ }^{4}$ Department of Disaster Psychiatry, School of Medicine, Fukushima Medical University, 1 Hikarigaoka, Fukushima-city, Fukushima 960-1295, Japan. ${ }^{5}$ Department of Gastroenterology, School of Medicine, Fukushima Medical University, 1 Hikarigaoka, Fukushima-city, 
Fukushima 960-1295, Japan. ${ }^{6}$ Department of Neuropsychiatry, School of Medicine, Fukushima Medical University, 1 Hikarigaoka, Fukushima-city, Fukushima 960-1295, Japan. 7 Department of Mental Health Policy, National Institute of Mental Health, National Center of Neurology and Psychiatry, 4-1-1 Ogawa-Higashi, Kodaira, Tokyo 187-8553, Japan.

Received: 11 June 2020 Accepted: 26 November 2020

Published online: 07 January 2021

\section{References}

1. Kunii Y, Suzuki Y, Shiga T, Yabe H, Yasumura S, Maeda M, Niwa S, Otsuru A Mashiko H, Abe M, Mental Health Group of the Fukushima Health Management Survey. Severe psychological distress of evacuees in evacuation zone caused by the Fukushima Daiichi nuclear power plant accident: The Fukushima Health Management Survey. PLoS One. 2016;11(7): e0158821.

2. Yabe H, Suzuki Y, Mashiko H, Nakayama Y, Hisata M, Niwa S, Yasumura S, Yamashita S, Kamiya K, Abe M, Mental Health Group of the Fukushima Health Management Survey. Psychological distress after the Great East Japan Earthquake and Fukushima Daiichi Nuclear Power Plant accident: results of a mental health and lifestyle survey through the Fukushima Health Management Survey in FY2011 and FY2012. Fukushima J Med Sci. 2014;60(1):57-67.

3. Maeda M, Oe M. Mental health consequences and social issues after the Fukushima disaster. Asia Pac J Public Health. 2017;29(2_suppl):36S-46S.

4. Kukihara H, Yamawaki N, Uchiyama K, Arai S, Horikawa E. Trauma, depression, and resilience of earthquake/tsunami/nuclear disaster survivors of Hirono, Fukushima Japan. Psychiatry ClinNeurosci. 2014;68(7):524-33.

5. Jiang T, Veres K, KörmendinéFarkas D, Lash TL, ToftSørensen H, Gradus JL. Post-traumatic stress disorder and incident fractures in the Danish population. OsteoporosInt. 2018;29(11):2487-93.

6. Ministry of Health, Labour and Welfare of Japan. Summary report of comprehensive survey of living conditions 2016. https://www.mhlw.go.jp/ english/database/db-hss/dl/report_gaikyo_2016.pdf. Accessed 14 July 2020.

7. Tanji F, Sugawara $Y$, Tomata $Y$, Watanabe T, Sugiyama K, Kaiho $Y$, Tomita H, Tsuji I. Psychological distress and the incident risk of functional disability in elderly survivors after the Great East Japan Earthquake. J Affect Disord. 2017; 221:145-50.

8. Yasumura S, Hosoya M, Yamashita S, Kamiya K, Abe M, Akashi M, Kodama K, Ozasa K, Fukushima Health Management Survey Group. Study protocol for the Fukushima Health Management Survey. J Epidemiol. 2012;22(5):375-83.

9. Hayashi F, Sanpei M, Ohira T, Nakano H, Okazaki K, Yasumura S, Nakajima S, Yabe H, Suzuki Y, Kamiya K, Fukushima Health Management Survey Group. Changes in the mental health status of adolescents following the Fukushima Daiichi nuclear accident and related factors: Fukushima Health Management Survey. J Affect Disord. 2020;260:432-9.

10. Radiation Medical Science Center for the Fukushima Health Management Survey, Fukushima Medical University. Mental Health and Lifestyle Survey (For Adults). https://fukushima-mimamori.jp/uploads/01_3.pdf. Accessed 16 Nov 2020

11. Blanchrd EB, Jones-Alexander J, Buckley TC, Forneris CA. Psychometric properties of the PTSD checklist (PCL). Behav Res Ther. 1996;34(8):669-73.

12. Iwasa $H$, Suzuki $Y$, Shiga $T$, et al. Psychometric evaluation of the Japanese version of the posttraumatic stress disorder checklist in community dwellers following the Fukushima Daiichi nuclear power plant incident: The Fukushima Health Management Survey. SAGE Open. 2016;6(2):1-11.

13. Suzuki Y, Yabe H, Horikoshi N, Yasumura S, Kawakami N, Ohtsuru A, Mashiko H, Maeda M, Mental Health Group of the Fukushima Health Management Survey. Diagnostic accuracy of Japanese posttraumatic stress measures after a complex disaster: The Fukushima Health Management Survey. Asia Pac Psychiatry. 2017;9(1):e12248.

14. Tsubokura M, Takita M, Matsumura T, Hara K, Tanimoto T, Kobayashi K, Hamaki T, Oiso G, Kami M, Okawada T, Tachiya H. Changes in metabolic profiles after the Great East Japan Earthquake: a retrospective observational study. BMC Public Health. 2013:13:267.

15. Satoh H, Ohira T, Hosoya M, Sakai A, Watanabe T, Ohtsuru A, Kawasaki Y, Suzuki H, Takahashi A, Kobashi G, Ozasa K, Yasumura S, Yamashita S, Kamiya K, Abe M. Evacuation after the Fukushima Daiichi nuclear power plant accident is a cause of diabetes: Results From the Fukushima Health Management Survey. J Diabetes Res. 2015;2015:627390.
16. Ohira T, Hosoya M, Yasumura S, Satoh H, Suzuki H, Sakai A, Ohtsuru A Kawasaki Y, Takahashi A, Ozasa K, Kobashi G, Kamiya K, Yamashita S, Abe M, Fukushima Health Management Survey Group. Effect of evacuation on body weight after the Great East Japan Earthquake. Am J Prev Med. 2016;50(5): 553-60.

17. Hashimoto $S$, Nagai M, Fukuma S, Ohira T, Hosoya M, Yasumura $S$, Satoh $H$, Suzuki H, Sakai A, Ohtsuru A, Kawasaki Y, Takahashi A, Ozasa K, Kobashi G, Kamiya K, Yamashita S, Fukuhara S, Ohto H, Abe M, Fukushima Health Management Survey Group. Influence of post-disaster evacuation on incidence of metabolic syndrome. J AtherosclerThromb. 2017;24(3):327-37.

18. Sakai A, Ohira T, Hosoya M, Ohtsuru A, Satoh H, Kawasaki Y, Suzuki H, Takahashi A, Kobashi G, Ozasa K, Yasumura S, Yamashita S, Kamiya K, Abe M, Fukushima Health Management Survey Group. Life as an evacuee after the Fukushima Daiichi nuclear power plant accident is a cause of polycythemia: The Fukushima Health Management Survey. BMC Public Health. 2014;14:1318.

19. Suzuki H, Ohira T, Takeishi Y, Hosoya M, Yasumura S, Satoh H, Kawasaki Y, Takahashi A, Sakai A, Ohtsuru A, Kobashi G, Ozasa K, Yamashita S, Kamiya K, Abe M, Fukushima Health Management Survey Group. Increased prevalence of atrial fibrillation after the Great East Japan Earthquake: results from the Fukushima Health Management Survey. Int J Cardiol. 2015;198:102-5.

20. Uemura M, Ohira T, Yasumura S, Otsuru A, Maeda M, Harigane M, Horikoshi N, Suzuki Y, Yabe H, Takahashi H, Nagai M, Nakano H, Zhang W, Hirosaki M, Abe M, Fukushima Health Management Survey Group. Association between psychological distress and dietary intake among evacuees after the Great East Japan Earthquake in a cross-sectional study: The Fukushima Health Management Survey. BMJ Open. 2016;6(7):e011534.

21. Zhang W, Ohira T, Abe M, Kamiya K, Yamashita S, Yasumura S, Ohtsuru A, Maeda M, Harigane M, Horikoshi N, Suzuki Y, Yabe H, Yuuki M, Nagai M, Takahashi H, Nakano H, Fukushima Health Management Survey Group. Evacuation after the Great East Japan Earthquake was associated with poor dietary intake: The Fukushima Health Management Survey. J Epidemiol. 2017;27(1):14-23.

22. Pedersen $A B$, Baggesen $L M$, Ehrenstein V, Pedersen $L$, Lasgaard M, Mikkelsen EM. Perceived stress and risk of any osteoporotic fracture. OsteoporosInt. 2016;27(6):2035-45.

23. Aardal-Eriksson E, Eriksson TE, Thorell LH. Salivary cortisol, posttraumatic stress symptoms, and general health in the acute phase and during 9month follow-up. Biol Psychiatry. 2001;50(12):986-93.

24. van Staa TP, Leufkens HG, Cooper C. The epidemiology of corticosteroidinduced osteoporosis: a meta-analysis. OsteoporosInt. 2002;13(10):777-87.

25. Oe M, Takahashi H, Maeda M, Harigane M, Fujii S, Miura I, Nagai M, Yabe H, Ohira T, Suzuki Y, Yasumura S, Abe M. Changes of posttraumatic stress responses in evacuated residents and their related factors. Asia Pac J Public Health. 2017;29(2_suppl):182S-192S

26. Maeda M, Oe M. The Great East Japan Earthquake: tsunami and nuclear disaster traumatic stress and long-term recovery. Traumatic stress and longterm recovery: coping with disasters and other negative life events. 2015. p. 71-90.

27. Kessler RC, Sonnega A, Bromet E, Hughes M, Nelson CB. Posttraumatic stress disorder in the National Comorbidity Survey. Arch Gen Psychiatry. 1995; 52(12):1048-60

28. Perkonigg A, Kessler RC, Storz S, Wittchen H-U. Traumatic events and posttraumatic stress disorder in the community: prevalence, risk factors and comorbidity. ActaPsychiatrScand. 2000;101(1):46-59.

29. Kessler RC, Andrews G, Colpe LJ, Hiripi E, Mroczek DK, Normand SL, Walters EE, Zaslavsky AM. Short screening scales to monitor population prevalences and trends in non-specific psychological distress. Psychol Med. 2002;32(6): 959-76.

30. Furukawa TA, Kawakami N, Saitoh M, Ono Y, Nakane $Y$, Nakamura $Y$, Tachimori H, Iwata N, Uda H, Nakane H, Watanabe M, Naganuma Y, Hata Y, Kobayashi M, Miyake Y, Takeshima T, Kikkawa T. The performance of the Japanese version of the K6 and K10 in the World Mental Health Survey Japan. Int J Methods Psychiatr Res. 2008;17(3):152-8.

31. Sakurai K, Nishi A, Kondo K, Yanagida K, Kawakami N. Screening performance of K6/K10 and other screening instruments for mood and anxiety disorders in Japan. Psychiatry PsychiatryClinNeurosci. 2011;65(5):434-41.

32. Yoshida K, Shinkawa T, Urata H, Nakashima K, Orita M, Yasui K, Kumagai A, Ohtsuru A, Yabe H, Maeda M, Hayashida N, Kudo T, Yamashita S, Takamura N. Psychological distress of residents in Kawauchi village, Fukushima Prefecture after the accident at Fukushima Daiichi Nuclear Power Station: the Fukushima Health Management Survey. PeerJ. 2016;4:e2353. 
33. Miura I, Nagai M, Maeda M, Harigane M, Fujii S, Oe M, Yabe H, Suzuki Y, Takahashi H, Ohira T, Yasumura S, Abe M. Perception of radiation risk as a predictor of mid-term mental health after a nuclear disaster: The Fukushima Health Management Survey. Int J Environ Res Public Health. 2017;14(9):1067.

34. Wu Q, Liu B, Tonmoy S. Depression and risk of fracture and bone loss: an updated meta-analysis of prospective studies. OsteoporosInt. 2018;29(6): 1303-12.

35. Wu Q, Liu J, Gallegos-Orozco JF, Hentz JG. Depression, fracture risk, and bone loss: a meta-analysis of cohort studies. OsteoporosInt. 2010;21(10): $1627-35$.

36. Wu Q, Bencaz AF, Hentz JG, Crowell MD. Selective serotonin reuptake inhibitor treatment and risk of fractures: a meta-analysis of cohort and casecontrol studies. OsteoporosInt. 2012;23(1):365-75.

37. Warden SJ, Fuchs RK. Do selective serotonin reuptake inhibitors (SSRIs) cause fractures? CurrOsteoporos Rep. 2016;14(5):211-8.

38. Doi Y, Minowa M, Okawa M, Uchiyama M. Prevalence of sleep disturbance and hypnotic medication use in relation to sociodemographic factors in the general Japanese adult population. J Epidemiol. 2000;10(2):79-86.

39. Wang PS, Bohn RL, Glynn RJ, Mogun H, Avorn J. Hazardous benzodiazepine regimens in the elderly: effects of half-life, dosage, and duration on risk of hip fracture. Am J Psychiatry. 2001;158(6):892-8.

40. Fonad E, Wahlin TB, Winblad B, Emami A, Sandmark H. Falls and fall risk among nursing home residents. J ClinNurs. 2008;17(1):126-34.

41. Fujiwara S, Kasagi F, Masunari N, Naito K, Suzuki G, Fukunaga M. Fracture prediction from bone mineral density in Japanese men and women. J Bone Miner Res. 2003;18(8):1547-53.

42. Riggs BL, Khosla S, Melton LJ 3rd. Sex steroids and the construction and conservation of the adult skeleton. Endocr Rev. 2002;23(3):279-302.

43. van der Hooft CS, Schoofs MW, Ziere G, Hofman A, Pols HA, Sturkenboom MC, Stricker BH. Inappropriate benzodiazepine use in older adults and the risk of fracture. Br J ClinPharmacol. 2008;66(2):276-82.

44. Brenneman SK, Barrett-Connor E, Sajjan S, Markson LE, Siris ES. Impact of recent fracture on health-related quality of life in postmenopausal women. J Bone Miner Res. 2006;21(6):809-16.

45. de Kam D, Smulders E, Weerdesteyn V, Smits-Engelsman BC. Exercise interventions to reduce fall-related fractures and their risk factors in individuals with low bone density: a systematic review of randomized controlled trials. OsteoporosInt. 2009;20(12):2111-25.

46. Vestergaard P, Mosekilde L. Fracture risk associated with smoking: a metaanalysis. J Intern Med. 2003;254(6):572-83.

47. Schwartz AV, Vittinghoff E, Bauer DC, Hillier TA, Strotmeyer ES, Ensrud KE, Donaldson MG, Cauley JA, Harris TB, Koster A, Womack CR, Palermo L, Black DM, Study of Osteoporotic Fractures (SOF) Research Group, Osteoporotic Fractures in Men (MrOS) Research Group, Health, Aging, and Body Composition (Health $\mathrm{ABC}$ ) Research Group. Association of BMD and FRAX score with risk of fracture in older adults with type 2 diabetes. JAMA. 2011 305(21):2184-92

48. Sennerby U, Melhus H, Gedeborg R, Byberg L, Garmo H, Ahlbom A, Pedersen NL, Michaëlsson K. Cardiovascular diseases and risk of hip fracture. JAMA. 2009:302(15):1666-73.

49. Kanis J, Oden A, Johnell O. Acute and long-term increase in fracture risk after hospitalization for stroke. Stroke. 2001;32(3):702-6.

50. Hadji P, Aapro MS, Body JJ, Bundred NJ, Brufsky A, Coleman RE, Gnant M, Guise T, Lipton A. Management of aromatase inhibitor-associated bone loss in postmenopausal women with breast cancer: practical guidance for prevention and treatment. Ann Oncol. 2011;22(12):2546-55.

51. Smith MR, Lee WC, Brandman J, Wang Q, Botteman M, Pashos CL. Gonadotropin-releasing hormone agonists and fracture risk: a claims-based cohort study of men with nonmetastatic prostate cancer. J ClinOncol. 2005; 23(31):7897-903.

52. Manabe J, Kawaguchi N, Matsumoto S, Tanizawa T. Surgical treatment of bone metastasis: indications and outcomes. Int J ClinOncol. 2005;10(2):103-11.

53. Oe M, Fujii S, Maeda M, Nagai M, Harigane M, Miura I, Yabe H, Ohira T, Takahashi H, Suzuki Y, Yasumura S, Abe M. Three-year trend survey of psychological distress, post-traumatic stress, and problem drinking among residents in the evacuation zone after the Fukushima Daiichi Nuclear Power Plant accident [The Fukushima Health Management Survey]. Psychiatry ClinNeurosci. 2016;70(6):245-52

54. Horikoshi N, Iwasa H, Yasumura S, Maeda M. The characteristics of nonrespondents and respondents of a mental health survey among evacuees in a disaster: The Fukushima Health Management Survey. Fukushima J Med Sci. 2017;63(3):152-9.

55. Kanis JA. Diagnosis of osteoporosis and assessment of fracture risk. Lancet. 2002;359(9321):1929-36.

56. Tanaka S, Kuroda T, Saito M, Shiraki M. Overweight/obesity and underweight are both risk factors for osteoporotic fractures at different sites in Japanese postmenopausal women. OsteoporosInt. 2013;24(1):69-76.

57. Kojima G. Frailty as a predictor of fractures among community-dwelling older people: a systematic review and meta-analysis. Bone. 2016;90:116-22.

58. Searle SD, Mitnitski A, Gahbauer EA, Gill TM, Rockwood K. A standard procedure for creating a frailty index. BMC geriatrics. 2008:8(1):24.

59. Yaegashi $Y$, Onoda T, Tanno K, et al. Association of hip fracture incidence and intake of calcium, magnesium, vitamin D, and vitamin K. Eur J Epidemiol. 2008;23(3):219-25.

60. Kojima A, Ikehara S, Kamiya K, et al. Natto intake is inversely associated with osteoporotic fracture risk in postmenopausal Japanese women. J Nutr. 2020; 150(3):599-605.

\section{Publisher's Note}

Springer Nature remains neutral with regard to jurisdictional claims in published maps and institutional affiliations.
Ready to submit your research? Choose BMC and benefit from:

- fast, convenient online submission

- thorough peer review by experienced researchers in your field

- rapid publication on acceptance

- support for research data, including large and complex data types

- gold Open Access which fosters wider collaboration and increased citations

- maximum visibility for your research: over $100 \mathrm{M}$ website views per year

At BMC, research is always in progress.

Learn more biomedcentral.com/submissions 